\title{
Eficacia histopatológica en la fase inflamatoria, proliferativa y osteogénica en la cicatrización alveolar post exodoncia aplicando Matico (Piper angustifolium)
}

\section{Histopathologic efficacy in inflammatory phase, proliferative and osteogenic healing in post extraction alveolar applying Matico (Piper angustifolium) \\ Cesar Cayo ${ }^{1}$, Aurelia Pérez ${ }^{2}$}

http://dx.doi.org/10.21503/CienciayDesarrollo.2014.V17i2.01

\section{RESUMEN}

El presente estudio tiene como finalidad determinar la eficacia histopatológica en la fase inflamatoria, proliferativa y osteogénica de la cicatrización post exodoncia tratadas con Matico (Piper angustifolium). El estudio fue experimental, tuvo por finalidad saber el nivel de cicatrización histopatológica del Matico (Piper angustifolium) dentro de los alveolos post exodoncia, con un grupo de estudio de 15 conejos machos con un peso de $2.5 \mathrm{Kg}$, divididos en grupo control y grupo experimental (se aplicó Matico), se realizó un estudio comparativo, se utilizó una ficha ad hoc para la recolección de resultados de la muestra, se aplicó el método estadístico, usando el programa estadístico SPSS versión 19.0. Para el análisis estadístico bivariado de los resultados se uso una prueba no paramétrica para variables cualitativas (chi cuadrado). Dando por resultado lo siguiente, a los 3 y 10 días post exodoncia se observó mayor recuento de polimorfonucleares, fibroblastos, fibras colágenas y neovasos en los alvéolos tratados con Matico respecto de los alvéolos control $(\mathrm{P}=0.096)$. A los 30 días se encontró mayor cantidad de trabéculas óseas en los alvéolos tratado con Matico a diferencia de los alvéolos control $(\mathrm{P}=0.135)$. Se observó una eficiencia relativa del Matico en la cicatrización alveolar; pero dicha eficiencia no es estadísticamente significativa. Obteniendo como resultado lo siguiente, en los 3, 10 y 30 días post exodoncia; se observó una eficiencia relativa del Matico en la cicatrización alveolar. Pero dicha eficiencia no es estadísticamente significativa; por ende, se rechaza la hipótesis de investigación.

Palabras claves: Matico (Piper angustifolium), cicatrización, conejo.

\section{ABSTRACT}

The present study aims to determine the effectiveness histopathological inflammatory phase, proliferative and osteogenic post extraction healing treated Matico (Piper angustifolium). The study was experimental was aimed to know the level of histopathological healing Matico (Piper angustifolium) within the post extraction sockets with a study group of 15 male rabbits weighing $2.5 \mathrm{~kg}$ were divided into control group and experimental group (Matico was applied), a comparative study was performed, an ad hoc collection of sample results sheet is used, the statistical method was applied, using SPSS version 19.0. For statistical analysis of the results bivariate nonparametric test was used for qualitative variables (chi -square). Resulting in the following, at 3 and 10 days post extraction polymorphonuclear count greater , fibroblasts, collagen fibers and alveoli neovessels treated with respect to the alveoli Matico control $(\mathrm{P}=$

\footnotetext{
1 Docente de Histología y Embriología de la EAP de Estomatología de la Facultad de Medicina Humana y Ciencias de la Salud de la UAP sede - Huacho,

E-mail: cesarcayorojas@gmail.com

2 Bachiller de la EAP de estomatología de la Facultad de Medicina Humana y Ciencias de la Salud de la UAP sede - Huacho.
} 
0.096 ) was observed. At 30 days increased trabecular bone amount was found in the cells treated with Matico alveoli unlike control $(\mathrm{P}=0.135)$. Matico relative efficiency in the alveolar healing was observed, but this efficiency is not statistically significant. Resulting in the following, in the 3,10 and 30 days post extraction, a relative efficiency on the alveolar Matico healing was observed. But this efficiency is not statistically significant; therefore, the research hypothesis is rejected.

Keywords: Matico (Piper angustifolium), scarring, rabbit.

\section{INTRODUCCIÓN}

El matico (Piper angustifolium) es una planta medicinal nativa, proveniente del sector andino, que se usa en heridas de tejidos blandos, ya que contiene una sustancia flavonoide que contribuyen a la cicatrización, lo cual se considera importante para la investigación.

Investigaciones recientes sobre esta planta medicinal (Piper angustifolium) como el de Guerrero D. en el año 2004 en la ciudad de Lima Perú, que comparó la reacción histológica del gel de matico versus la pasta convencional de Óxido de Zinc Eugenol, en el proceso de cicatrización en alveolitis seca inducida en conejos. Donde se obtuvo como resultado en todas las muestras histológicas, que el gel de matico ofrecía mejores resultados que los tratados con pasta de Óxido de Zinc Eugenol. Con lo que se concluyó, que el gel de matico en el proceso de cicatrización, manifiesta mejores resultados, principalmente en la epitelización, resorción ósea y osteogénesis en comparación del grupo que se trató con Óxido de Zinc Eugenol. (1)

En el año 2004 Kishimoto T. en la ciudad de Lima Perú realizo un estudio donde comparó el efecto de un apósito periodontal con y sin matico en el proceso de cicatrización del tejido conectivo de la mucosa palatina en conejos, según etapas. Este estudio se llevó acabo en 14 conejos machos de raza california de 3 meses de edad de 2 a $2.5 \mathrm{~kg}$ de peso, todos aparentemente sanos. Se procedió a hacer una incisión en cada hemiarcada, a nivel de las rugas palatinas, dejando expuesto el tejido conectivo, donde se aplicó, el apósito con matico en uno de los bordes laterales y el otro solo se suturó. El estudio se realizó en tres etapas a las 24 horas, 3 días y 7 días. Donde se obtuvo mejor eficacia de cicatrización histológica el grupo tratado con apósito de matico. (2)

En el año 2010, Menéndez C. en la ciudad de Arequipa Perú, realizó un estudio clínico comparativo de la efectividad del gel de matico (Piper angustifolium) en la evolución de la cicatrización de heridas de la mucosa bucal post exodoncia del tercer molar inferior incluido, sobre un fármaco que controle el proceso de cicatrización de una herida operatoria. Obteniendo como resultado que el gel de matico es efectivo frente a los indicadores: Sangrado, edema y unión de los bordes de la herida operatoria. Con lo cual se concluye, que el gel de matico, es una herramienta útil, para conseguir mejores resultados en el periodo post operatorio de exodoncias de piezas incluidas. (3)

En el año 2012 Muñoz C, et al. En la ciudad de Viña del mar Chile, realizaron un estudio donde se seleccionaron cuatro plantas utilizadas por la medicina mapuche debido a sus propiedades cicatrizantes: tallo de nalca, corteza de tineo y hojas de matico y canelo. El objetivo fue identificar en las plantas seleccionadas la presencia de compuestos químicos que favorecen los procesos de cicatrización y evaluar su capacidad antioxidante. La presencia de compuestos fenólicos se realizó mediante reacciones fotoquímicas. Se identificaron diversos tipos de flavonoides y taninos en los 
extractos de las plantas evaluadas, siendo en el caso de la corteza del tineo y matico donde se encontraron dichos compuestos. En relación a la actividad antioxidante, destacan la actividad demostrada por los extractos de tineo y nalca al ser comparados con vitamina C. (4)

Otros autores como Vázquez J et al en el año 2013 Universidad de Chile, compararon los cambios histológicos en la reparación de una herida en paladar de rata tratada con un preparado de hojas de matico y llantén incluidos en plastibase, respecto a un control solo con plastibase y a uno sin tratamiento. Todos los procedimientos fueron aprobados por el Comité de Ética de los Animales de la Facultad de Ciencias Químicas y Farmacéuticas. Se procedió a realizar una lesión estándar de $3 \mathrm{~mm}$ de diámetro, en el paladar de 36 ratas Sprague Dawley divididas en tres grupos tratadas con: 1) Plastibase más preparado herbal, 2) Plastibase solo y 3) Sin tratamiento. Se controló clínicamente por 7 días. Donde se obtuvo mejores características histológicas en el grupo tratado con extracto herbal (matico, llantén) con una aceleración en el proceso inflamatorio, fibroplasia, angiogénesis y maduración de colágeno, así como un menor número de mastocitos. Con lo que se concluyó que el preparado de matico y llantén en plastibase acelera el proceso de reparación de ulcera palatina de rata respecto a los controles. (5)

Estos antecedentes nos llevaron a la siguiente interrogante ¿Cuál es el nivel de eficacia histopatológica en la fase inflamatoria, proliferativa y osteogénica en la cicatrización alveolar post exodoncia aplicando matico (Piper angustifolium)? A lo cual se buscó la respuesta a esta pregunta a través del siguiente objetivo Determinar la eficacia histopatológica en la fase inflamatoria, proliferativa y osteogénica de la cicatrización post exodoncia aplicando matico (Piper angustifolium).

\section{MATERIALES Y MÉTODOS}

El estudio se desarrolló en la ciudad de Huacho durante los meses de junio a octubre del 2013; la población estuvo conformada por 15 conejos de raza California, machos en buen estado general y nutricional (diagnosticado por el especialista) con una edad de 6 meses y con un peso promedio de entre $2.5 \mathrm{~kg}-3 \mathrm{~kg}$.

El estudio fue experimental que tuvo por finalidad saber el nivel de cicatrización histopatológica del matico (Piper angustifolium) dentro de los alveolos post exodoncia, la muestra fue dividida en grupo control y grupo experimental (se aplicó matico), se realizó un estudio comparativo, se utilizó una ficha ad hoc para la recolección de resultados de la muestra, se aplicó el método estadístico, usando el programa estadístico SPSS versión 19.0.

En el manejo del experimento se consideró dos variables de estudio: una variable independiente, constituida por un producto natural, el matico (Piper angustifolium), la variable dependiente, los niveles de cicatrización histopatológica post exodoncia en la fase inflamatoria, proliferativa y osteogénica que fueron operacionalizadas en cuatro niveles para cada uno.

A todos los especímenes se le realizó examen bucal y una profilaxis antes de la cirugía. Se realizó el control de los signos vitales, comprobando el estado de inconsciencia del espécimen se procedido a la fase quirúrgica. Todos los especímenes fueron sometidos a una extracción dental en la zona anterior a nivel superior e inferior por los operadores, previa capacitación por un (Cirujano dentista).

Una vez extraída las piezas dentarias, al grupo No 01 se aplicó directamente el matico (Piper angustifolium) hasta rellenar el alvéolo superior y se procedió a suturar. Seguidamente al grupo 
No 02 no se le aplicó medicamento alguno y se procedió a suturar con Vicryl (RB1-5/0).

Se sacrificó a los especímenes por asfixia con éter y se obtuvo las muestras de acuerdo al tiempo experimental: 5 muestras a los 3 días post exodoncia; a los 10 días post exodoncia y a los 30 días post exodoncia.

A los animales sacrificados se le removió la mandíbula y se separaron ambas hemiarcadas, las cuales se fijaron en formol al $10 \%$ por 24 horas, luego descalcificadas en ácido nítrico al $5 \%$ por 36 horas y sometidas al procedimiento de rutina para obtener muestras coloreadas con hematoxilina-eosina. Los cortes histológicos fueron observadas con un microscopio óptico compuesto de marca Carls zeiss, que fueron analizadas por un especialista en patología oral y maxilofacial, con más de 10 años de experiencia ,quien evaluó a simple ciego los siguientes indicadores: polimorfonucleares, fibroblastos, fibras colágenas y neovasos (a los 3dias); fibroblastos, fibras colágenas y neovasos (a los 10 días); neovasos, células osteogénicas, espículas óseas y trabéculas óseas (a los 30 días). El estudio histopatológico se hizo a nivel de la mitad apical del alvéolo.

Por último se procedió a anotar los resultados en una ficha ad hoc. Por el mismo patólogo y posteriormente se procedo a realizar el análisis estadístico asesorado por un experto especialista en tema con más de diez años de experiencia.

\section{RESULTADOS}

Gráfico 1: Nivel de eficacia histopatológica en la cicatrización a los 3 días post exodoncia, en fase inflamatoria. Y proloferativa recuento de polimorfonucleares

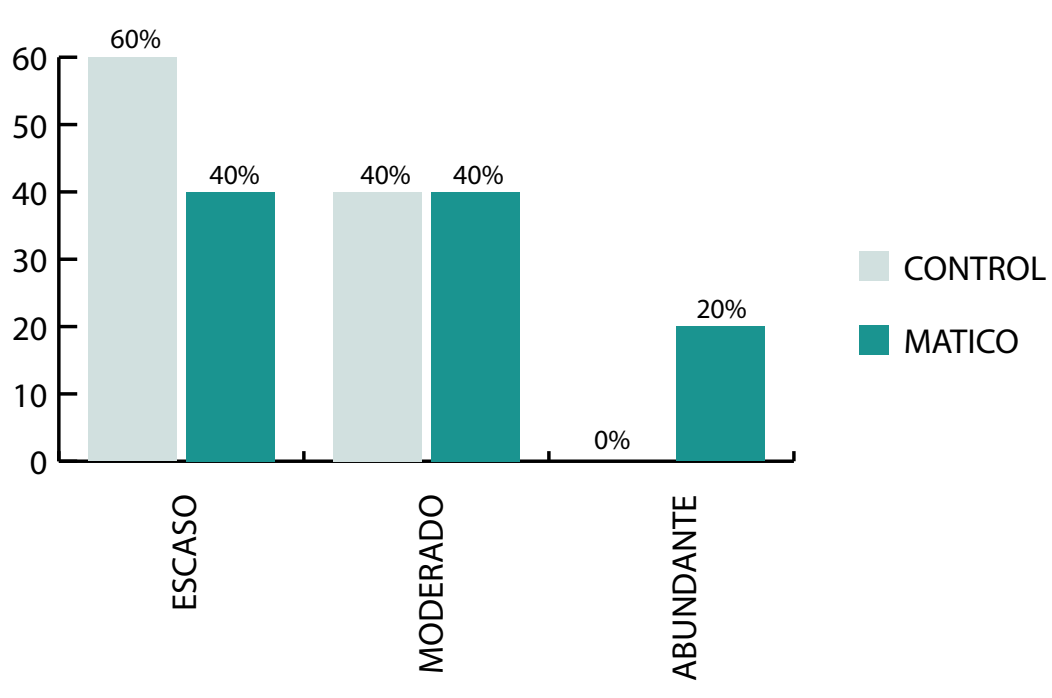

RECUENTO DE POLIMORFONUCLEARES
Se ha encontrado mayor cantidad de polimorfonucleares en el alveolo tratado con matico; es decir, existe una diferencia absoluta y relativa entre el alveolo tratado con matico y el alveolo control. Pero dicha diferencia no es estadísticamente significativa. 
Gráfico 2: Nivel de eficacia histopatológica en la cicatrización a los 3 días post exodoncia, en fase inflamatoria y proloferativa. Recuento de fibroblastos

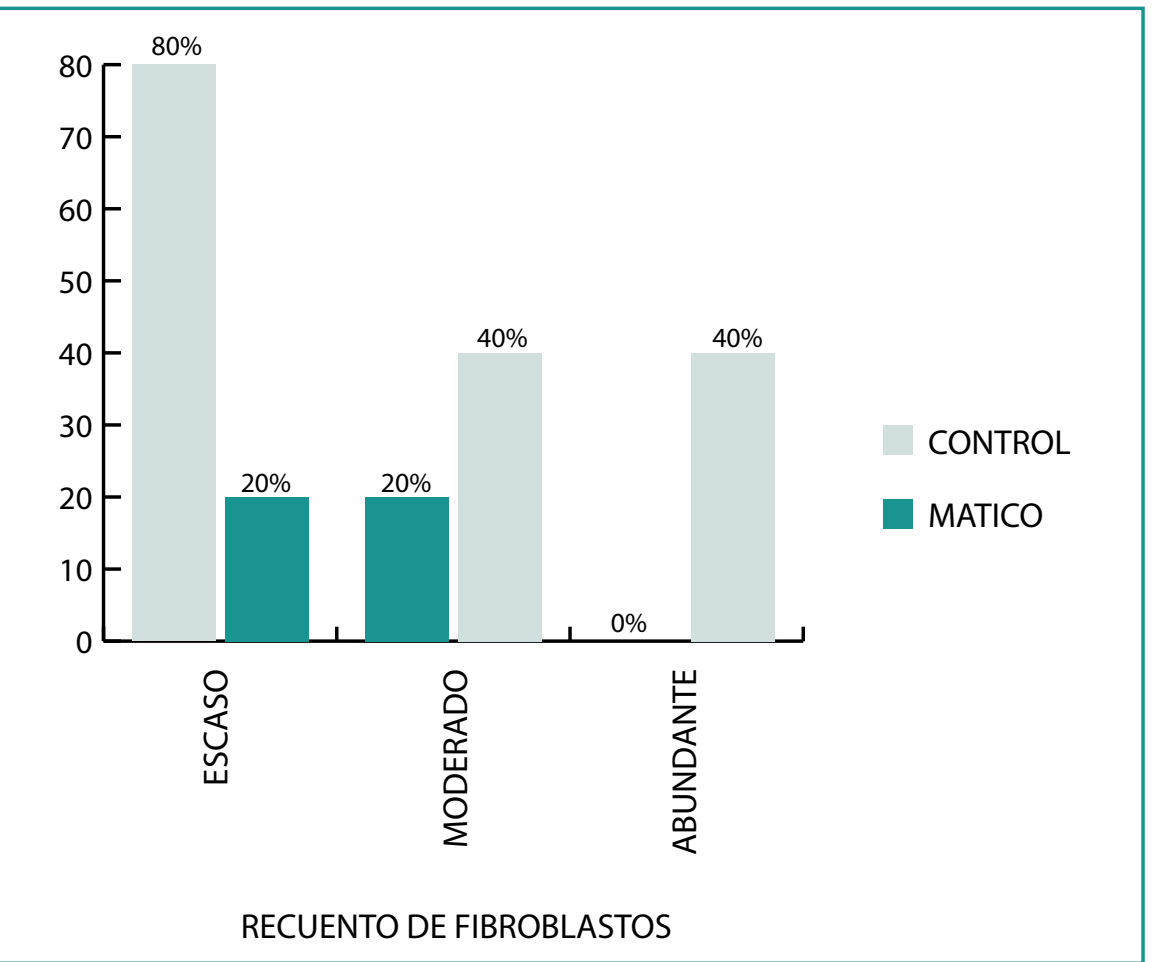

Se ha encontrado mayor cantidad de fibroblastos en el alveolo tratado con matico; es decir, existe una diferencia absoluta y relativa entre el alveolo tratado con matico y el alveolo control. Pero dicha diferencia no es estadísticamente significativa.

Gráfico 3: Nivel de eficacia histopatológica en la cicatrización a los 3 días post exodoncia, en fase inflamatoria y proloferativa. Recuento de fibras colagenas

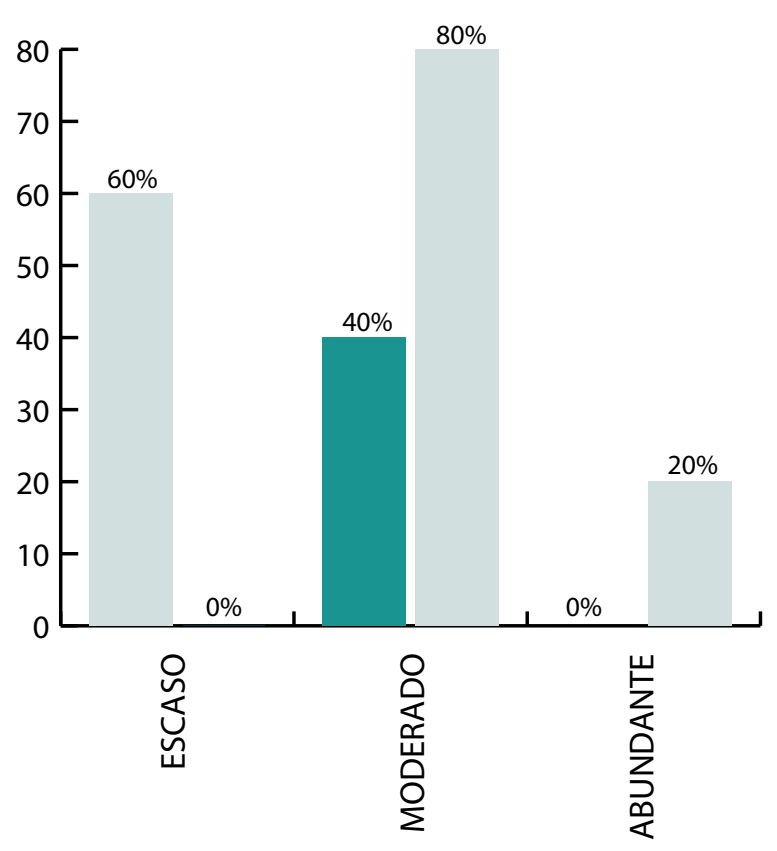

CONTROL

MATICO
Se ha encontrado mayor cantidad de fibras colágenas en el alveolo tratado con matico; es decir, existe una diferencia absoluta y relativa entre el alveolo tratado con matico y el alveolo control. Pero dicha diferencia no es estadísticamente significativa. 
Gráfico 4: Nivel de eficacia histopatológica en la cicatrización a los 3 días post exodoncia, en fase inflamatoria y proloferativa. Recuento de neovasos

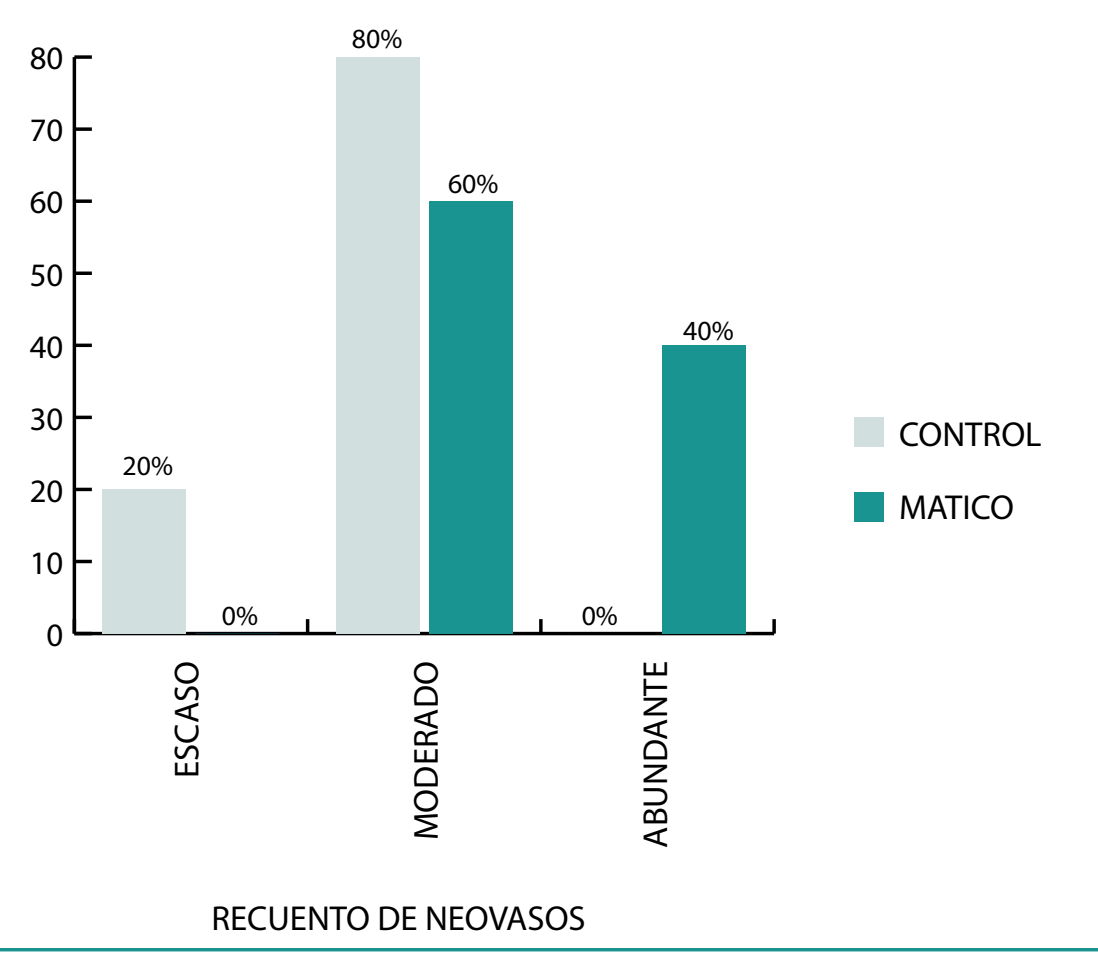

Se ha encontrado mayor cantidad de neovasos en el alveolo tratado con matico; es decir, existe una diferencia absoluta y relativa entre el alveolo tratado con matico y el alveolo control. Pero dicha diferencia no es estadísticamente significativa.

Gráfico 5: Nivel de eficacia histopatológica en la cicatrización a los 10 días post exodoncia, en fase inflamatoria y proliferativa. Recuento de fibroblastos

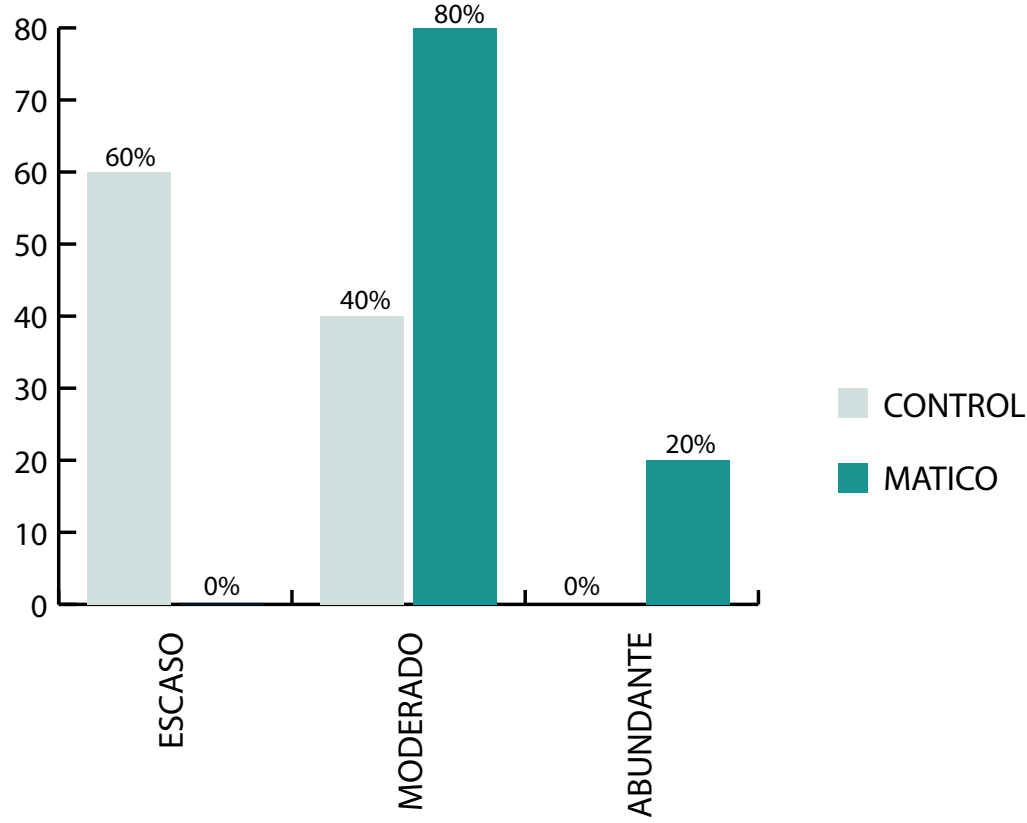

RECUENTO DE FIBROBLASTOS
Se ha encontrado mayor cantidad de fibroblastos en el alveolo tratado con matico; es decir, existe una diferencia absoluta y relativa entre el alveolo tratado con matico y el alveolo control. Pero dicha diferencia no es estadísticamente significativa. 
Gráfico 6: Nivel de eficacia histopatológica en la cicatrización a los 10 días post exodoncia, en fase inflamatoria y proloferativa. Recuento de fibras colágenas

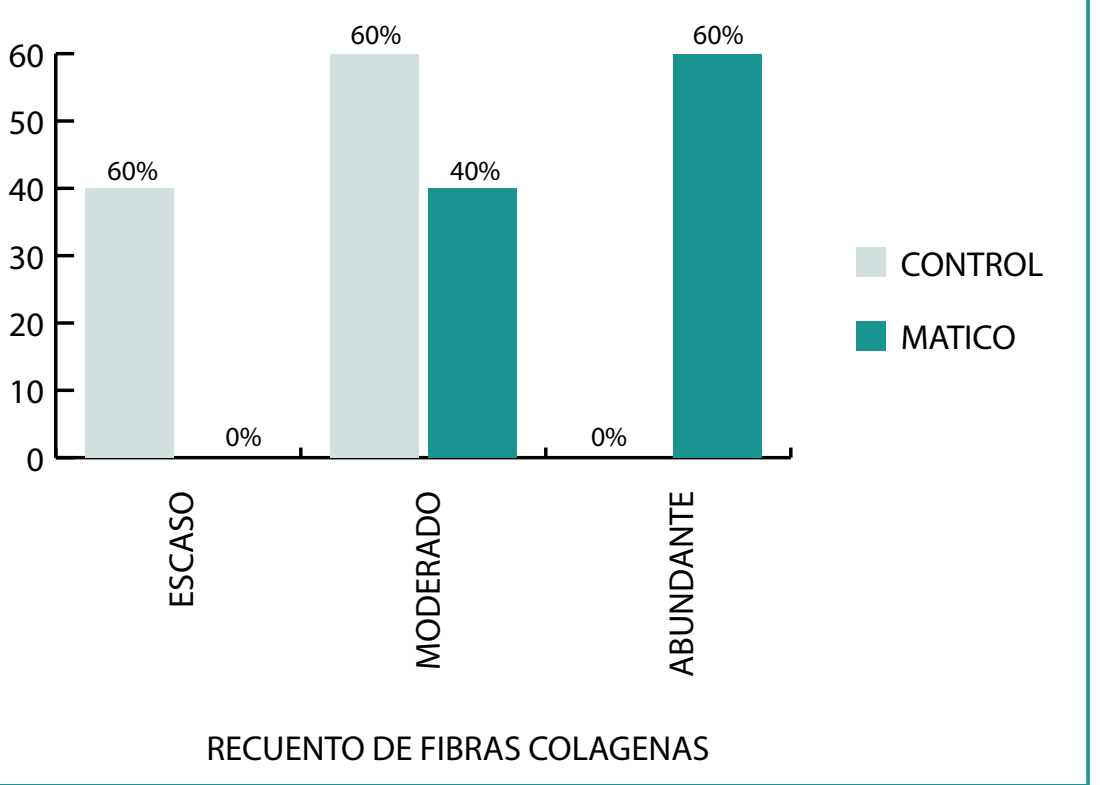

Se ha encontrado mayor cantidad de fibras colágenos en el alveolo tratado con matico; es decir, existe una diferencia absoluta y relativa entre el alveolo tratado con matico y el alveolo control. Pero dicha diferencia no es estadísticamente significativa.

Gráfico 7: Nivel de eficacia histopatológica en la cicatrización a los 10 días post exodoncia, en fase inflamatoria y proloferativa. Recuento de neovasos

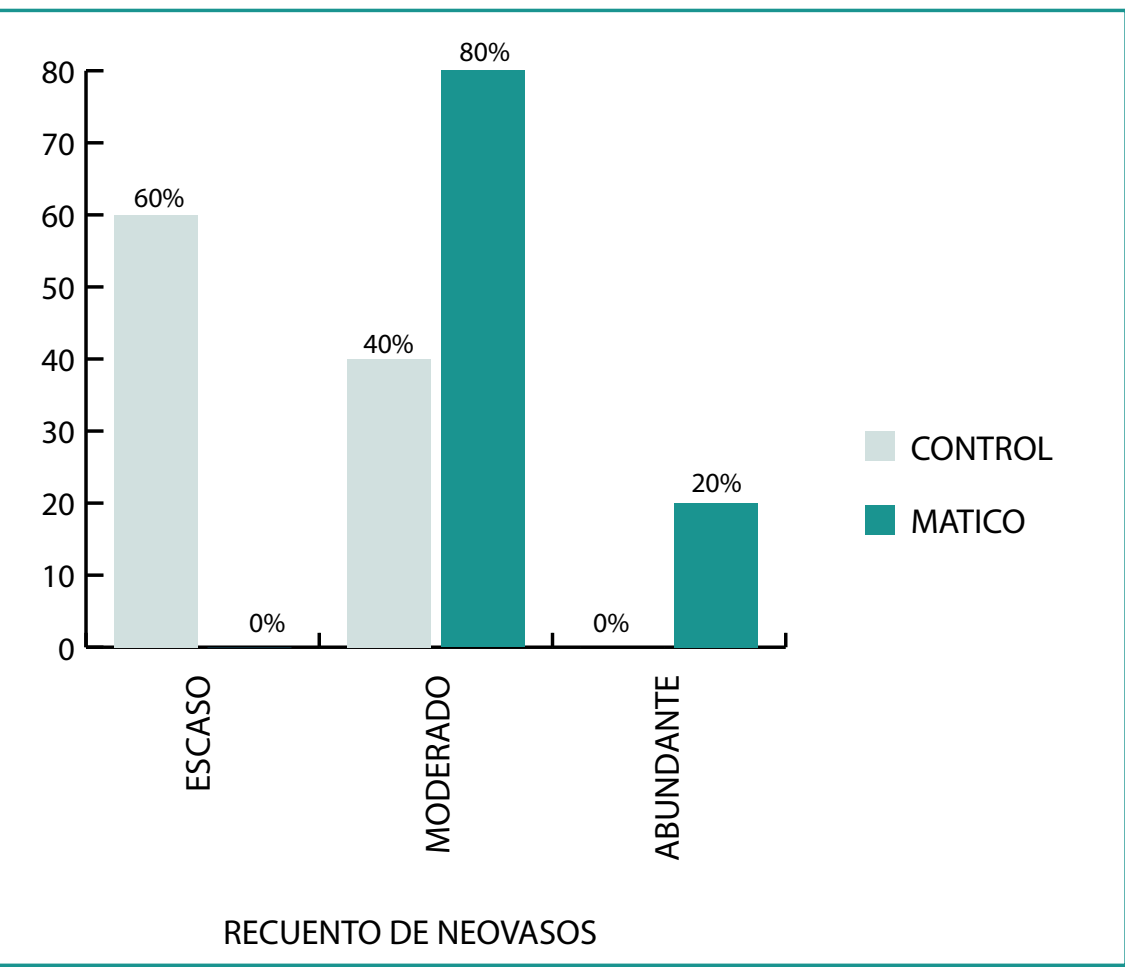

Se ha encontrado mayor cantidad de neovasos en el alveolo tratado con matico; es decir, existe una diferencia absoluta y relativa entre el alveolo tratado con matico y el alveolo control. Pero dicha diferencia no es estadísticamente significativa. 
Gráfico 8: Nivel de eficacia histopatológica en la cicatrización a los 30 días post exodoncia, en fase osteogénica. Recuento de neovasos

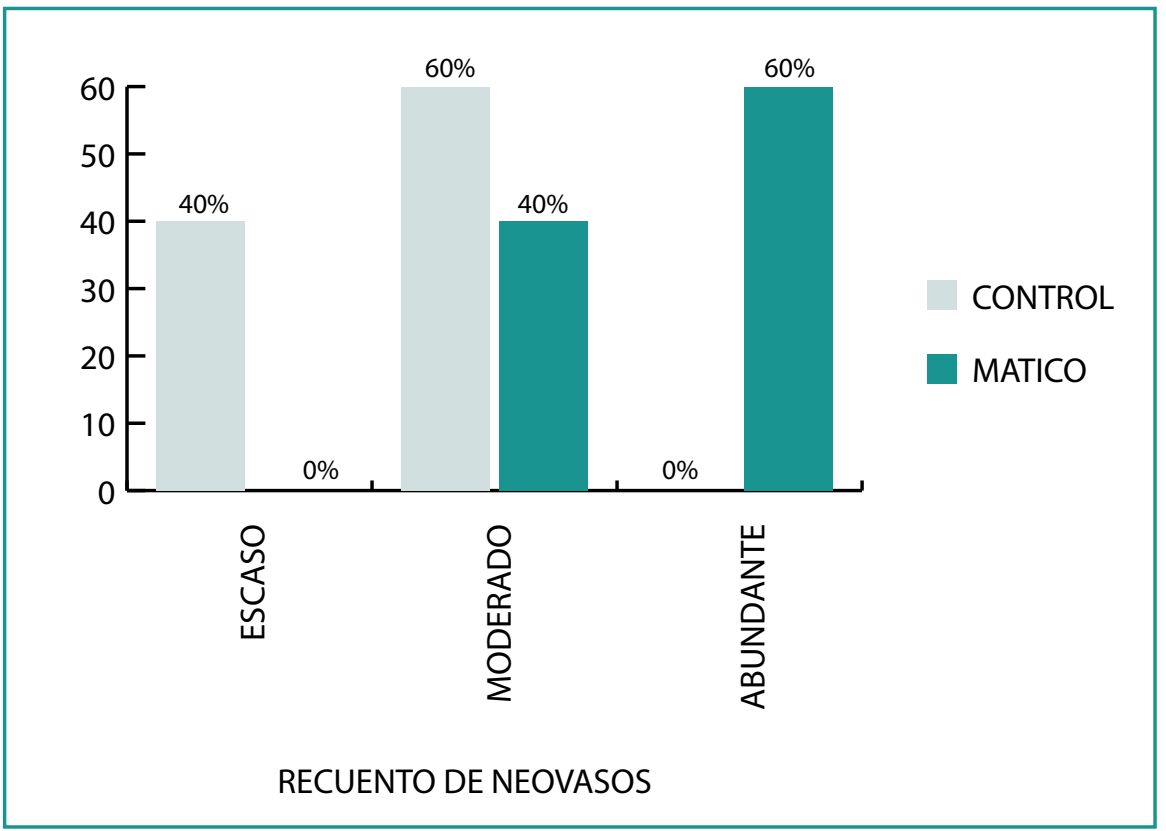

Se ha encontrado mayor cantidad de neovasos en el alveolo tratado con matico; es decir, existe una diferencia absoluta y relativa entre el alveolo tratado con matico y el alveolo control. Pero dicha diferencia no es estadísticamente significativa.

Gráfico 9: Nivel de eficacia histopatológica en la cicatrización a los 30 días post exodoncia, en fase osteogénica. Recuento de células osteogénicas

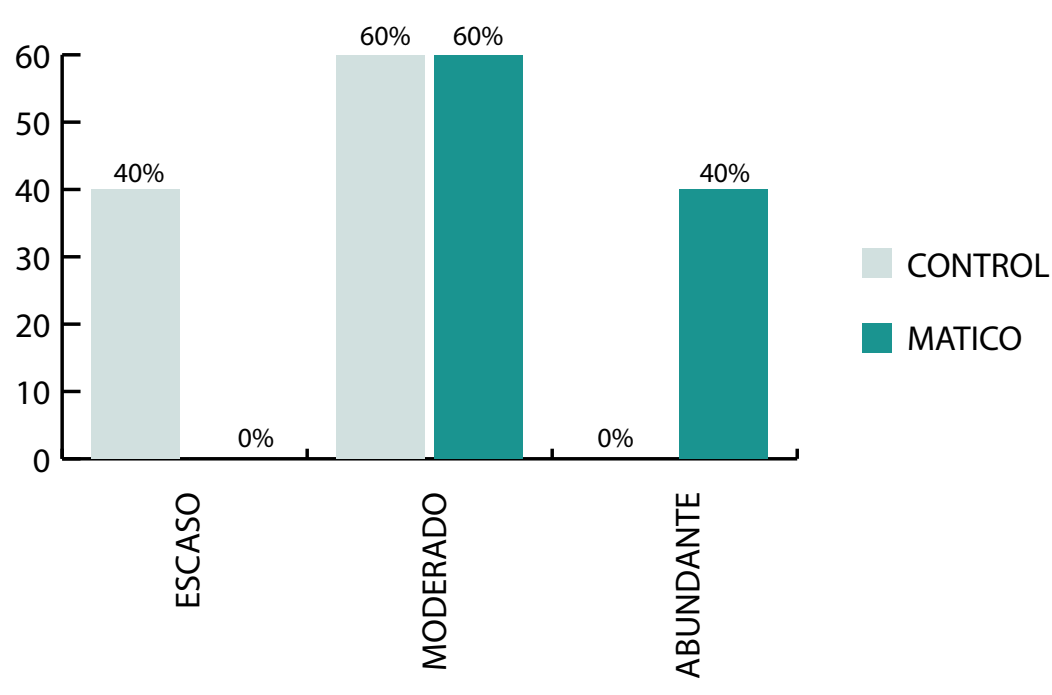

RECUENTO DE CÉLULAS OSTEOGÉNICAS
Se ha encontrado mayor cantidad de células osteogénicas en el alveolo tratado con matico; es decir, existe una diferencia absoluta y relativa entre el alveolo tratado con matico y el alveolo control. Pero dicha diferencia no es estadísticamente significativa. 
Gráfico 10: Nivel de eficacia histopatológica en la cicatrización a los 30 días post exodoncia, en fase osteogénica. Recuento de espículas óseas

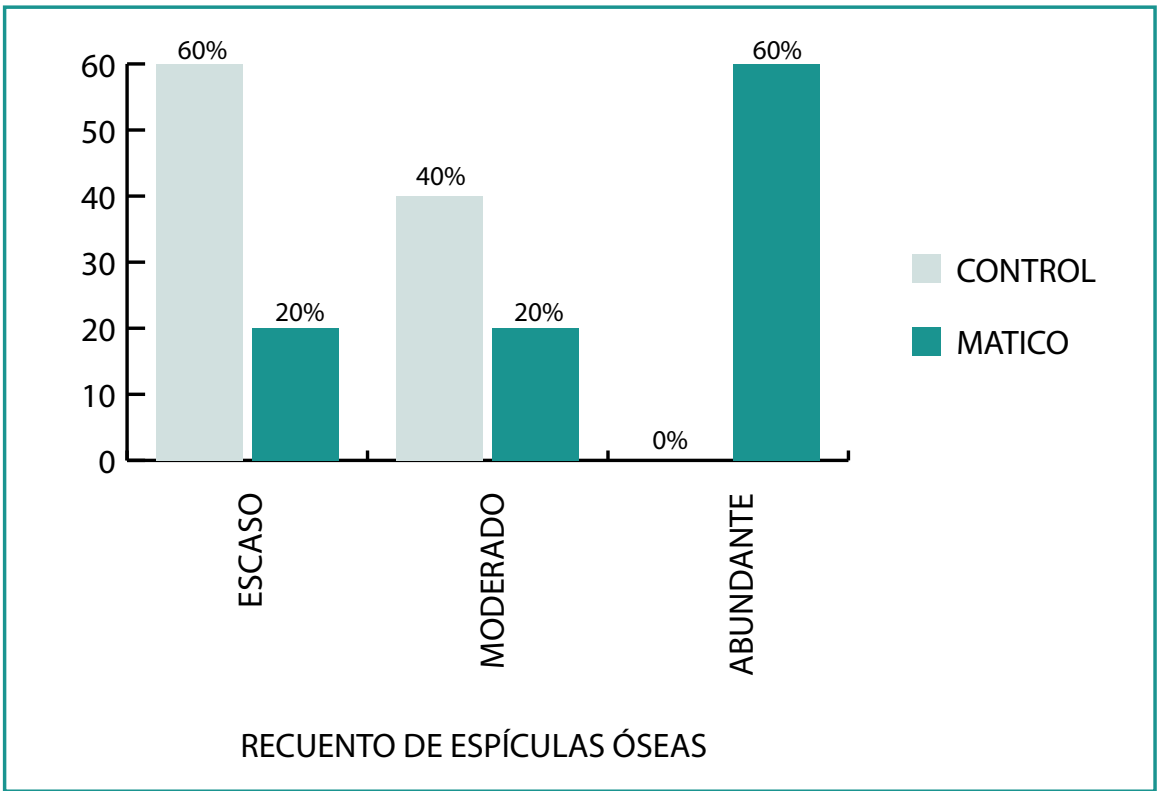

Se ha encontrado mayor cantidad de espículas óseas en el alveolo tratado con matico; es decir, existe una diferencia absoluta y relativa entre el alveolo tratado con matico y el alveolo control. Pero dicha diferencia no es estadísticamente significativa.

Gráfico 11: Nivel de eficacia histopatológica en la cicatrización a los 30 días post exodoncia, en fase osteogénica. Recuento de trabéculas óseas

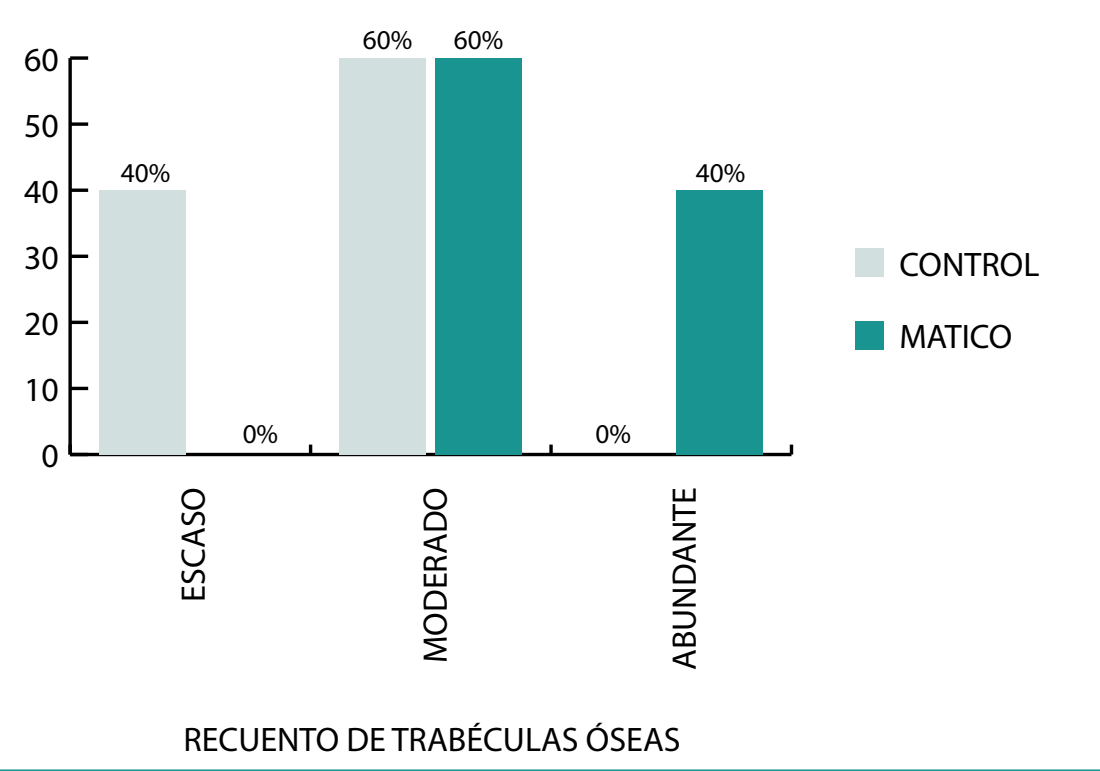

Se ha encontrado mayor cantidad de trabéculas óseas en el alveolo tratado con matico; es decir, existe una diferencia absoluta y relativa entre el alveolo tratado con matico y el alveolo control. Pero dicha diferencia no es estadísticamente significativa. 


\section{CONCLUSIONES}

A los tres días post exodoncia se hizo una evaluación histopatológica en fase inflamatoria y proliferativa. Se encontró mayor recuento de polimorfonucleares, fibroblastos, fibras colágenas y neovasos en los alveolos tratados con matico respecto de los alveolos control. El matico potencia la cicatrización a los tres días; pero esta potenciación no es significativa.

A los diez días post exodoncia se hizo una evaluación histopatológica en fase inflamatoria y proliferativa. Se encontró mayor recuento de fibroblastos, fibras colágenas y neovasos en los alveolos tratados con matico respecto de los alveolos control. El matico potencia la cicatrización a los diez días; pero esta potenciación no es significativa.

A los treinta días post exodoncia se hizo una evaluación histopatológica en fase osteogénica. Se encontró mayor recuento de polimorfonucleares, fibroblastos, fibras colágenas y neovasos en los alveolos tratados con matico respecto de los alveolos control. El matico potencia la osteogénesis a los treinta días; pero esta potenciación no es significativa.

En los 3, 10 y 30 días post exodoncia; se observó una eficiencia relativa del matico en la cicatrización alveolar. Pero dicha eficiencia no es estadísticamente significativa; por ende, se rechaza la hipótesis de investigación.

\section{DISCUSIÓN}

Se ha encontrado mayor cantidad de células polimorfonucleares en el alvéolo tratado con matico; es decir, existe una diferencia absoluta y relativa entre el alveolo tratado con matico y el alveolo control. Pero dicha diferencia no es estadísticamente significativa en comparación al estudio realizado por Guerrero D en el año 2004 (1) que comparó la reacción histológica del gel de matico versus la pasta convencional de Óxido de Zinc Eugenol, en el proceso de cicatrización en alveolitis seca inducida en conejos, donde se concluyó, que el gel de matico en el proceso de cicatrización, manifiesta mejores resultados, principalmente en la epitelización, resorción ósea y osteogénesis en comparación del grupo que se trató con Óxido de Zinc Eugenol.

El matico potencia la cicatrización a los 3 días, 10 días y 30 días, pero esta potenciación no es significativa. En comparación al estudio realizado Kishimoto T en el año 2004 (2), que comparó el efecto de un apósito periodontal con y sin matico en el proceso de cicatrización del tejido conectivo de la mucosa palatina en conejos, según etapas. El estudio se realizó en tres etapas a las 24 horas, 3 días y 7 días, donde se obtuvo mejor eficacia de cicatrización histológica el grupo tratado con apósito de matico.

El matico potencia la cicatrización a los 3 días 10 días y 30 días a nivel histológico pero dicha efectividad no es estadísticamente significativa en comparación al estudio realizado por Menéndez C en el año 2010 (3), que realizó un estudio clínico comparativo de la efectividad del gel de matico en la evolución de la cicatrización de heridas de la mucosa bucal post exodoncia del tercer molar inferior incluido, sobre un fármaco que controle el proceso de cicatrización de una herida operatoria. Obteniendo como resultado que el gel de matico es efectivo frente a los indicadores: Sangrado, edema y unión de los bordes de la herida operatoria.

\section{AGRADECIMIENTOS}

A los estudiantes de segundo ciclo de la escuela profesional de estomatología que formaron parte de este estudio por su valiosa colaboración. 


\section{REFERENCIAS BIBLIOGRÁFICAS}

1. Guerrero D. Gel de matico vs. la pasta convencional de Óxido de Zinc Eugenol en el proceso de cicatrización en alveolitis seca inducido en Oniclotagus cuniculus [Tesis para optar el título de Cirujano dentista]. Lima: Universidad Federico Villareal, Facultad de Odontología; 2004.

2. Kishimoto T. Efecto de un apósito periodontal con y sin matico en el proceso de cicatrización en la mucosa palatina de conejos. [Tesis para optar el título de Cirujano dentista]. Lima: Universidad Federico Villareal, facultad de Odontología; 2004.

3. Menéndez C. Efectividad del gel de matico (Piper angustifolium) en la evolución de la cicatrización de heridas de la mucosa bucal post exodoncia del tercer molar inferior incluido en el Hospital Nacional Carlos Alberto Seguin Escobedo Arequipa 2010 [Tesis para optar el título de Cirujano Dentista]. Arequipa: Universidad de Arequipa. Facultad de Odontología; 2010.

4. Muñoz C, et al. Investigación de extractos de plantas medicinales usadas por sus propiedades cicatrizantes. Ciencia Joven. 27 de julio de 2012;(1):59-62.

5. Vázquez J, et al. Aspectos Histológicos de un Preparado Medicinal en Reparación de Herida Palatina Estandarizada en Ratas. V Encuentro Científico de Estudiantes de Odontología de la Universidad de Chile. 2013. 